\title{
A 6-YEAR RETROSPECTIVE ANALYSIS ON THE SPECIES DISTRIBUTION AND ANTIMICROBIAL SUSCEPTIBILITY OF PATHOGENS IN BLOOD CULTURES
}

\author{
Qing-Yong Wang, Rong-Hai Li, Bei Song, Xiao-Hong Shang* \\ Department of Clinical Laboratory, Xiyuan Hospital, China \\ Academy of Chinese Medical Sciences, Beijing, 100091, China
}

\begin{abstract}
It is important for microbiological workers to perform periodic surveillances on the emergence of isolates from positive blood cultures and their antibiotic resistance. The aim of this study is to evaluate the common pathogenic distribution and antimicrobial susceptibility of pathogens isolated from aerobic and anaerobic bottles for the past six years. From January 2010 to December 2015, 9122 bottles were tested using an automated blood culture detection system. From 9122 bottles, 745 positive bottles yielded 450 organisms. Of these, 250 (55.6\%) were Gram-negative bacteria, 184 (40.9\%) were Gram-positive bacteria and 16 (3.6\%) were fungi. Escherichia coli and Staphylococcus spp were the most frequently isolated species for Gram-negative and Gram-positive bacteria, respectively. Amikacin, meropenem, imipenem, vancomycin, linezolid and quinupristin-dalfopristin may be the best choices to use for positive blood cultures in the hospital. The periodic surveillance of the common pathogenic distribution isolated from bottles and their antimicrobial susceptibilities can help medical workers to recognize the emergence and rapid dissemination of pathogens with high rates of resistance to most commonly used antibiotics.
\end{abstract}

Key Words: Blood culture; Species distribution; Antimicrobial susceptibility.

\section{INTRODUCTION}

Bacterial bloodstream infections can lead to the high morbidity and mortality of people globally. Thus, it is important for laboratories to detect pathogens on time and identify these pathogens using an optimal blood culture system and technology to initiate the right antimicrobial therapy. Accurate and timely blood culture results are essential for medical workers to provide appropriate antibiotic therapy for patients with sepsis or septicemia [1]. Most medical institutions rely on an automated blood culture system to detect blood pathogens. In our hospital, no studies have analyzed the distribution of pathogens and the antimicrobial susceptibility of these pathogens from commercially available BACTEC blood bottles. Therefore, we conducted this study.

\section{Materials and methods}

A retrospective study was conducted in the Department of Clinical Laboratory. Data analysis was carried out between January 2010 and December 2015. A total of 745 positive blood culture samples were examined by our lab.

The culture bottles were incubated using the BACTEC 9240 blood culture system. A blood culture set (consisting of one resin-containing BACTEC Plus

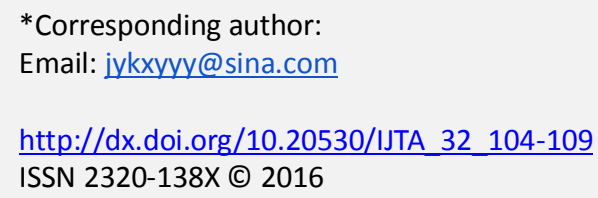

Aerobic/F and one BACTEC Lytic/10 Anaerobic/F) were incubated at $35^{\circ} \mathrm{C}$ with continuous rocking agitation for 5 days, and tested by the system unit every ten minutes. The bottles were discarded if no microbial growth was detected by the fluorescence technology system (over 5 days). The parts of the bottles flagged as positive were used for Gram staining and subcultured on blood agar plates and China blue agar plates for further identification. Bacterial identification and antimicrobial susceptibility of the isolated pathogens were performed using the BD phoenix Automated Microbiology System.

Each bloodstream infection was defined as the isolation of a unique organism from one, two, or more bottles collected within 7 days along with clinical evidence of infection, the repeated isolation of the same pathogen after 7 days of the last known positive culture, or a pathogen which was different from the initial pathogen. If more than two culture bottles from the same patient were positive, only one bottle was used for the antimicrobial susceptibility assay. Reference strains of Escherichia Coli (ATCC 25922), Pseudomonas aeruginosa (ATCC 27853), Enterococcus faecalis (ATCC 29212) and Staphylococcus aureus (ATCC 29213) were used as quality control strains according to the manufacturer's instructions. Additional tests were conducted if the yielded results of the BD Phoenix Automated Microbiology System had $<90 \%$ confidence.

\section{RESULTS}

During the study period, a total of 9122 blood 
bottles were submitted to the lab, and 745 (8.2\%) positive bottles (445 aerobic bottles and 300 anaerobic bottles) yielded 450 organisms in this study. Of the patients $(n=426)$ with positive blood cultures, 168 (39.4\%) were females and 258 (60.6\%) were males, with a mean age of 69.8 years (range 19-99 years). Among the 450 isolates, 402 isolates (89.3\%) were single species. Of the 450 isolates, 250 (55.6\%) were Gram-negative bacteria, 184 (40.9\%) were Gram-positive bacteria and 16 (3.6\%) were fungi. The ten most common isolates accounted for $351(78 \%)$ of all the isolates. Of these, 155 isolates from bottles ( $\geq 1$ set blood culture) were analyzed, and 137 were detected in the aerobic bottles only and 59 were from anaerobic bottles only.

As shown in Table 1, Escherichia Coli was the predominant Gram-negative bacteria followed by Klebsiella pneumoniae, Pseudomonas aeruginosa, Acinetobacter baumannii and Enterobacter cloacae. The five leading pathogens classified as Gram-positive bacteria were Staphylococcus epidermidi, Staphylococcus aureus, Enterococcus faecalis, Enterococcus faecium, and Staphylococcus haemolyticus. All isolates of $A$. baumannii were observed in aerobic bottles. For those positive bottles with $P$. aeruginosa and $S$. haemolyticus isolates, only a few isolates grew in anaerobic bottles compared to that of aerobic bottles, and no isolates of $P$. aeruginosa and $S$. haemolyticus grew only in anaerobic bottles. Furthermore, compared to $E$. faecium, the isolates of $E$. faecalis grew more in anaerobic bottles. Almost all the isolates of $C$. albicans were observed in the aerobic bottles, and only one isolate grew in an anaerobic bottle (data not shown). We also found that nine isolates were obligate anaerobes (eight Gram-negative and one Gram-positive). Table 2 and Table 3 show the antimicrobial susceptibility test results performed during the study period. Higher amikacin, meropenem and imipenem susceptibilities were found of more than $77.1 \%, 61.5 \%$ and $69.2 \%$ respectively among the four Gram-negative bacteria isolates tested except for A. baumannii isolates. Only aztreonam displayed the highest susceptibility (54.2\%) for A. baumannii. Vancomycin, linezolid and quinupristin/dalfopristin susceptibility remained high $(79.8 \%, 84.2 \%$ and $89.5 \%)$ for the five leading pathogens in Gram-positive bacteria respectively, and no resistance were found to vancomycin and linezolid among the three Staphylococcus spp.

\section{DISCUSSION}

The blood culture system has improved the ability to continuously monitor positive bottles as soon as possible, including the addition of various bottles type, advances in the agitation system, and the development of related software. The rapid detection and identification of pathogens from blood culture bottles is important for lab workers. At the same time, empirical antimicrobial therapy has been shown to have the greatest impact on patients outcomes, so we need to provide information on the common pathogenenic distribution and antimicrobial susceptibility in a timely manner. In our study, a retrospective survey was conducted on 745 positive blood culture samples from the microbiology lab between January 2010 and December 2015. In this study, a blood culture set consists of one resin-containing BACTEC Plus Aerobic/F bottle and one BACTEC Lytic/10 Anaerobic/F bottle. The BACTEC Aerobic Plus resin bottle contains resin which bind and neutralize a wide variety of antimicrobial agents, although it may occasionally not do well for some antibiotics [2]. The

Table1. Top of 10 pathogens detected in BACTEC blood culture bottles. (2010.1.1 - 2015.12.31)

\begin{tabular}{|c|c|c|c|c|c|c|c|}
\hline Pathogen & $\begin{array}{l}\text { No. of } \\
\text { isolates }\end{array}$ & $\begin{array}{c}\text { Growth in } \\
\text { aerobic } \\
\text { bottles only }\end{array}$ & $\begin{array}{l}\text { Growth in } \\
\text { anaerobic } \\
\text { bottles only }\end{array}$ & $\begin{array}{l}\text { Growth in } \\
\text { both } \\
\text { bottles } \\
\text { ( } \geq 1 \text { culture) }\end{array}$ & $\begin{array}{l}\text { No. of } \\
\text { positive } \\
\text { bottles }\end{array}$ & $\begin{array}{c}\text { Growth in } \\
\text { aerobic } \\
\text { bottles }\end{array}$ & $\begin{array}{c}\text { Growth in } \\
\text { anaerobic } \\
\text { bottles }\end{array}$ \\
\hline E.coli & 95 (44.2\%) & 25 & 21 & 49 & 158 & 82 & 76 \\
\hline K. pneumoniae & $48(22.3 \%)$ & 14 & 8 & 26 & 98 & 50 & 48 \\
\hline P. aeruginosa & $31(14.4)$ & 24 & 0 & 7 & 39 & 31 & 8 \\
\hline A. baumannii & $28(13 \%)$ & 28 & 0 & 0 & 37 & 37 & 0 \\
\hline E. cloacae & $13(6 \%)$ & 4 & 1 & 8 & 24 & 14 & 10 \\
\hline Total & 215 & 95 (44.2\%) & 30 (14\%) & 90 (41.9\%) & 356 & $214(60.1 \%)$ & 142 (39.9\%) \\
\hline S. epidermidis & 47 (34.6\%) & 14 & 9 & 24 & 75 & 40 & 35 \\
\hline E. faecalis & $26(19.1 \%)$ & 1 & 10 & 15 & 61 & 26 & 35 \\
\hline S. aureus & $25(18.4 \%)$ & 7 & 8 & 10 & 40 & 21 & 19 \\
\hline E. faecium & $19(14 \%)$ & 7 & 2 & 10 & 36 & 20 & 16 \\
\hline $\begin{array}{c}\text { S. } \\
\text { haemolyticus }\end{array}$ & $19(14 \%)$ & 13 & 0 & 6 & 25 & 19 & 6 \\
\hline Total & 136 & 42 (30.9\%) & 29 (21.3\%) & 65 (47.8\%) & 237 & $126(53.2 \%)$ & $111(46.8 \%)$ \\
\hline
\end{tabular}


Table 2. Antimicrobial susceptibility pattern of the top five Gram negative bacterial isolates from blood culture.

\begin{tabular}{cccccc}
\hline E.coli(N=95) & $\begin{array}{c}\mathbf{K} . \\
\text { pneumoniae } \\
(\mathbf{N = 4 8 )}\end{array}$ & $\begin{array}{c}\mathbf{P} \text { a aeruginosa } \\
\mathbf{( N = 3 1 )}\end{array}$ & $\begin{array}{c}\text { A. baumannii } \\
\mathbf{( N = 2 8 )}\end{array}$ & $\begin{array}{c}\text { E. cloacae } \\
\mathbf{( N = 1 3 )}\end{array}$ \\
\hline AN & $93.7 \%$ & $77.1 \%$ & $100 \%$ & $25 \%$ & $84.6 \%$ \\
AMC & $41 \%$ & $47.9 \%$ & 0 & 0 & 0 \\
AM & $9.5 \%$ & 0 & 0 & 0 & 0 \\
ATM & $42.1 \%$ & $54.2 \%$ & $61.3 \%$ & $53.6 \%$ & $38.5 \%$ \\
CIP & $26.3 \%$ & $58.3 \%$ & $87.1 \%$ & $14.3 \%$ & $53.8 \%$ \\
MEM & $98.9 \%$ & $79.2 \%$ & $90.3 \%$ & $25 \%$ & $61.5 \%$ \\
PIP & $12.6 \%$ & $33.3 \%$ & $77.4 \%$ & 0 & $30.8 \%$ \\
TZP & $85.3 \%$ & $50 \%$ & $87.1 \%$ & $14.3 \%$ & $46.2 \%$ \\
GM & $47.4 \%$ & $58.3 \%$ & $90.3 \%$ & $17.9 \%$ & $46.2 \%$ \\
TE & $28.4 \%$ & $60.4 \%$ & 0 & $21.4 \%$ & $53.8 \%$ \\
FEP & $42.1 \%$ & $54.2 \%$ & $64.5 \%$ & $10.7 \%$ & $61.5 \%$ \\
CTX & $35.8 \%$ & $52.1 \%$ & 0 & $3.6 \%$ & $38.5 \%$ \\
CAZ & $50.5 \%$ & $56.3 \%$ & $71 \%$ & $17.9 \%$ & $46.2 \%$ \\
IPM & $97.9 \%$ & $79.2 \%$ & $77.4 \%$ & $25 \%$ & $69.2 \%$ \\
LVX & $28.4 \%$ & $66.7 \%$ & $77.4 \%$ & $17.9 \%$ & $53.8 \%$ \\
\hline
\end{tabular}

AN, Amikacin; AMC, Amoxicillin/Clavulanate; AM, Ampicillin; ATM, Aztreonam; CIP, Ciprofloxacin; MEM, Meropenem; PIP, Piperacillin; TZP, Piperacillin/Tazobactam; GM, Gentamicin; TE, Tetracycline; FEP, Cefepime; CTX, Cefotaxime; CAZ, Ceftazidime; IPM, Imipenem; LVX, Levofloxacinzuo.

BACTEC Lytic/10 Anaerobic/F bottle could destroy red blood cells, white blood cells and platelets, while at the same time release bacteria from circulating macrophages, increase positive detection rate, and shorten the reporting time. For some strains, the recovery was faster from anaerobic lytic bottles than aerobic resin-containing bottles [3]. Paired culture sets are recommended according to the Clinical and Laboratory Standards Institute guidelines [2].

In our study, a total of 745 positive bottles were identified which included 445 aerobic bottles and 300 anaerobic bottles. Almost all the isolates of $A$. baumannii, P. aeruginosa, S. haemolyticus, $C$. albicans, B. cepacia, S. maltophilia, O. anthropi, and Cedecea. spp were detected only in aerobic bottles. It was not surprising that these bottles with detected growth contained aerobic medium because that they are generally obligate aerobes. The isolates of $S$. lugdunensis, S. equorum, S. sciuri, S. constellatus, S. oralis, and $S$. pasteuri were detected only in anaerobic bottles. Despite a few uncommon strains in our data, such as one S. maltophilia, other studies have shown that its mortality rate was high as a marker of serious pathology [4]. In addition, it was noteworthy that in this study there were 24 cases where more than one pathogen was isolated from one or more blood culture bottles.

As shown in Table 1, the ratio of Gram-negative isolates to Gram-positive isolates was about 1.6:1. E. coli $(44.2 \%)$, and $K$. pneumoniae $(22.3 \%)$ were the more frequently isolated species for Gram-negative bacteria. This finding supports the results observed by other studies [5-6]. In addition, $S$. epidermidis (34.9\%) was the most frequently isolated species for Gram-positive bacteria. This finding supports the prior observation that $S$. epidermidis was the leading pathogen in early-onset sepsis [6]. All patients with isolates of coagulase-negative Staphylococcus should be evaluated by physicians to determine whether or not their real bloodstream was infected [7]. Our results had a higher proportion of Staphylococcus aureus among the Gram-positive bacteria. This bacteria is a common and most lethal cause of blood stream infections [8].

It was also interesting to find that the number of aerobic bottles was larger than that of the anaerobic bottles for the ten most common bacteria, except for $E$. faecalis. This indicates the unique characteristics of blood culture for $E$. faecalis. High numbers of facultative anaerobes were detected in the anaerobic and aerobic bottles. Using both bottles might have an important effect on the clinical therapy outcome [9].

Table 2 shows that there was higher amikacin, meropenem and imipenem susceptibility among the four Gram-negative bacteria isolates tested except for $A$. baumannii isolates. We found that there was a $100 \%$ susceptibility of amikacin for $P$. aeruginosa. The $A$. baumannii isolates showed high resistance for most antibiotics, and only aztreonam displayed the 
Table 3. Antimicrobial susceptibility pattern of the top five Gram Postive bacterial isolates from blood culture.

\begin{tabular}{cccccc}
\hline S. epidermidis & $\begin{array}{c}\mathbf{E} \text { faecalis } \\
(\mathbf{N}=\mathbf{2 6})\end{array}$ & $\begin{array}{c}\text { S. aureus } \\
(\mathbf{N}=\mathbf{2 5})\end{array}$ & $\begin{array}{c}\text { E. faecium } \\
(\mathbf{N}=\mathbf{1 9})\end{array}$ & $\begin{array}{c}\text { S. haemolyticus } \\
(\mathbf{N}=\mathbf{1 9})\end{array}$ \\
\hline AM & 0 & $88.5 \%$ & 0 & 0 & 0 \\
GMX & NA & $38.5 \%$ & NA & $47.4 \%$ & NA \\
LZD & $100 \%$ & $88.5 \%$ & $100 \%$ & $84.2 \%$ & $100 \%$ \\
VA & $100 \%$ & $92.3 \%$ & $100 \%$ & $78.9 \%$ & $100 \%$ \\
CIP & $31.9 \%$ & NA & $48 \%$ & NA & $10.5 \%$ \\
E & $23.4 \%$ & NA & $24 \%$ & NA & $5.3 \%$ \\
GM & $36.2 \%$ & NA & $40 \%$ & NA & $15.8 \%$ \\
OX & $6.4 \%$ & NA & $40 \%$ & NA & $10.5 \%$ \\
P & 0 & NA & 0 & NA & 0 \\
RA & $87.2 \%$ & NA & $56 \%$ & NA & $89.5 \%$ \\
SXT & $29.8 \%$ & NA & $76 \%$ & NA & $57.9 \%$ \\
SYN & $85.1 \%$ & NA & $96 \%$ & NA & $89.5 \%$ \\
TE & $68.1 \%$ & NA & $44 \%$ & NA & $73.7 \%$ \\
\hline
\end{tabular}

AM, Ampicillin; GMX, Gentamicin-syn; LZD, Linezolid; VA, Vancomycin; CIP, Ciprofloxacin; E, Erythromycin; GM, Gentamicin; OX, Oxacillin; P, Penicllin; RA, Rifampin; SXT, Trimethoprim/Sulfamethoxazole; SYN, Quinupristin/Dalfopristin; TE, Tetracycline; NA, not applicable.

highest susceptibility (54.2\%).

As shown in Table 3, there were higher susceptibilities towards vancomycin, linezolid, quinupristin/dalfopristin and lower susceptibility towards penicillin among the three Staphylococcus spp. In addition, the two Enterococcus spp had higher susceptibilities towards vancomycin and linezolid. Nevertheless, it was noteworthy to compare the rates of ampicillin susceptibilities for $E$. faecalis and the other four most common Gram-positive isolates ( $88.5 \%$ vs. $0 \%$ ) in our findings. The main findings of the present study was that vancomycin, linezolid, and quinupristin/dalfopristin were effective for Gram-positive bacteria, while amikacin, meropenem and imipenem were effective for Gram-negative bacteria. The antibody susceptibility pattern of isolates were different from the results reported by other studies [10]. Therefore, the in vitro susceptibility of this study highlights that these six antibiotics would be effective for the treatment of bloodstream infections in this area.

There are several potential limitations of this study. Information about the early availability of microbiological information (such as Gram stain findings, time of detection and so on) should be discussed briefly, which would be of enormous value in improving the outcome of severely infected patients. This is because timeliness and accuracy of preliminary blood culture reports are core laboratory quality performance indicators in the practice of assessing blood cultures [11].

Another limitation in our study which has to be pointed out is the incubation time for the Candida species. Although the Candida species rank lower among the pathogens isolated from the blood cultures in our study and little information was gained, we must consider the question of whether we should prolong the incubation time, perform terminal subcultures, or utilize specific mycology blood bottles for the detection of certain pathogens when using the BACTEC 9240 system. This is because we noted that for $C$. glabrata, the time to detection with the automated blood culture system was significantly longer in the aerobic medium than in the anaerobic medium, and was also longer than for other Candida spp. in aerobic mediums [12]. Compared to C. albicans, C. parapsilosis, C. tropicalis and $C$. krusei, a time-to-positivity of $\leq 56.5 \mathrm{~h}$, growth only or earlier in aerobic bottles would be useful to rule out $C$. glabrata for early treatment optimization as reported by other studies [13]. Despite these shortcomings, our data are critically important and could perhaps facilitate physicians to perform the initiation of appropriate empiric antibiotic treatment to some degree.

In conclusion, loading culture bottles into the instrument promptly is recommended in the microbiology laboratories of most hospitals. Good lab facilities, optimal testing parameters, phlebotomy team strategies or sterile collection kits are necessary to help medical workers have good guidelines for antibiotic use, during the empirical, early, or late periods [14-16]. Blood cultures remain a reliable and most practical method in the diagnosis of bloodstream infection, but other accurate and 
cost-effective methods are also used in clinical microbiology lab for rapid identification. One example is matrix-assisted laser desorption ionization-time of flight mass spectrometry, used especially for the rapid identification of Gram-negative Bacilli from blood cultures to influence the selection of the right antimicrobial therapy [17-18]. Although less of a gold standard regular diagnostic procedure, the PCR method could be valuable when used complementarily for bone marrow transplants with bloodstream infections [19] In addition, peptide nucleic acid fluorescent in situ hybridization (PNA-FISH) is also a possible method for the detection of species directly from positive blood culture bottles and peritoneal fluid cultures, in spite of a requirement to have more than $10^{5}$ $\mathrm{CFU} / \mathrm{mL}$ for detected organisms [20].

It is important for those in clinical medicine to perform blood cultures to detect pathogens and guide antibiotic therapy. ${ }^{21}$ Lab workers would have to consider adjusting the protocols accordingly because of several potential problems, including the timing of collection and loading the bottles into the instrument, type of culture bottle, longer time to detection with small inoculum sizes, and unusual growth characteristics of some pathogens, one shift per microbiology laboratory, the prior use of antibiotics, best practices (venipuncture and the use of phlebotomy teams), and repeat blood culture despite an initial negative blood culture with febrile neutropenia patients $[1,2,9,12,22-24]$. So it is important for both lab workers and physicians to understand the advantage, limitations and possible solutions for various automated blood culture system technologies, and perform oral-alert or written reports with good clinical advice $[16,25]$. The physician could request longer durations when clinically indicated.

With an increasing incidence of blood pathogens (including Candida species isolates), lab workers should also consider prolonging the incubation time of the blood culture, performing terminal subcultures before discarding, or using specific media for some pathogens based on the actual situation. Our study highlights that it is important for microbiological workers to perform periodic surveillances on the emergence of isolates from positive blood cultures and their antibiotic resistance.

\section{ACKNOWLEDGEMENTS}

None

\section{REFERENCES}

1. Snyder SR, Favoretto AM, Baetz RA, Derzon JH, Madison BM, Mass D, et al. Effectiveness of practices to reduce blood culture contamination: A Laboratory Medicine Best Practices systematic review and meta-analysis. Clinical Biochemistry. 2012 Sep;45(13-14):999-1011. Available from: http://dx.doi.org/10.1016/j.clinbiochem.2012.06.007

2. Towns ML, Jarvis WR, Hsueh P-R. Guidelines on Blood Cultures. Journal of Microbiology, Immunology and Infection. 2010 Aug;43(4):347-9. Available from: http://dx.doi.org/10.1016/s1684-1182(10)60054-0.

3. Horvath LL, Hospenthal DR, Murray CK, Dooley DP. Detection of Simulated Candidemia by the BACTEC 9240 System with Plus Aerobic/F and Anaerobic/F Blood Culture Bottles. Journal of Clinical Microbiology. 2003 Oct 1;41(10):4714-7. Available from: http://dx.doi.org/10.1128/jcm.41.10.4714-4717.2003

4. Araoka $H$, Baba $M$, Yoneyama A. Risk factors for mortality among patients with Stenotrophomonas maltophilia bacteremia in Tokyo, Japan, 1996-2009. European Journal of Clinical Microbiology \& Infectious Diseases. 2010 Feb 23;29(5):605-8. Available from: http://dx.doi.org/10.1007/s10096-010-0882-6.

5. Shanthachol T, Nilgate $S$, Suankratay $C$. A comparative study to determine the recovery rate of microorganisms of bloodstream infections: two versus three blood culture specimens. J Med Assoc. 2012 Aug; 95(8): 1053-8. Available from: http://www.jmatonline.com/index.php/jmat/article/v iew/2030.

6. Li Z, Xiao Z, Li Z, Zhong Q, Zhang Y, Xu F. 116 cases of neonatal early-onset or late-onset sepsis: A single center retrospective analysis on pathogenic bacteria species distribution and antimicrobial susceptibility. Int J Clin Exp Med. 2013 Sep 1; 6(8):693-9. Available from: http://www.ijcem.com/V6_No8.html.

7. Uyanik MH, Yazgi H, Ozden K, Erdil Z, Ayyildiz A. Comparison of Coagulase-Negative Staphylococci Isolated from Blood Cultures as a True Bacteremia Agent and Contaminant in Terms of Slime Production and Methicillin Resistance. EAJM. 2014 Jun 21;46(2):115-9. Available from: http://dx.doi.org/10.5152/eajm.2014.26.

8. Paulsen J, Mehl A, Askim Å, Solligård E, Åsvold B, Damås J. Epidemiology and outcome of Staphylococcus aureus bloodstream infection and sepsis in a Norwegian county 1996-2011: an observational study. BMC Infectious Diseases [Internet]. Springer Science + Business Media; 2015;15(1):116. Available from: http://dx.doi.org/10.1186/s12879-015-0849-4.

9. Janapatla RP, Yan J-J, Chien M-L, Chen H-M, Wu H-M, Wu J-J. Effect of Overnight Storage of Blood Culture Bottles on Bacterial Detection Time in the BACTEC 9240 Blood Culture System. Journal of Microbiology, Immunology and Infection. 2010 Apr;43(2):126-32. Available from: http://dx.doi.org/10.1016/s1684-1182(10)60020-5.

10. Wasihun AG, Wlekidan LN, Gebremariam SA, Dejene 
TA, Welderufael AL, Haile TD, et al. Bacteriological profile and antimicrobial susceptibility patterns of blood culture isolates among febrile patients in Mekelle Hospital, Northern Ethiopia. Springerplus. 2015 Jul 3; 4:314. Available from: http://springerplus.springeropen.com/articles/10.118 6/s40064-015-1056-x.

11. Schifman RB, Meier FA, Souers RJ. Timeliness and Accuracy of Reporting Preliminary Blood Culture Results: A College of American Pathologists Q-Probes Study of 65 Institutions. Archives of Pathology \& Laboratory Medicine. 2015 May;139(5):621-6. Available from: http://dx.doi.org/10.5858/arpa.2014-0258-cp.

12. George BJ, Horvath LL, Hospenthal DR. Effect of Inoculum Size on Detection of Candida Growth by the BACTEC 9240 Automated Blood Culture System Using Aerobic and Anaerobic Media. Journal of Clinical Microbiology. 2005 Jan 1;43(1):433-5. Available from: http://dx.doi.org/10.1128/jcm.43.1.433-435.2005.

13. Cobos-Trigueros N, Morata L, Torres J, Zboromyrska Y, Soriano A, Pitart C, et al. Usefulness of time-to-positivity in aerobic and anaerobic vials to predict the presence of Candida glabrata in patients with candidaemia. Journal of Antimicrobial Chemotherapy. 2013 Jul 9;68(12):2839-41. Available from: http://dx.doi.org/10.1093/jac/dkt285.

14. Self WH, Talbot TR, Paul BR, Collins SP, Ward MJ. Cost Analysis of Strategies to Reduce Blood Culture Contamination in the Emergency Department: Sterile Collection Kits and Phlebotomy Teams. Infection Control. 2014 Aug;35(08):1021-8. Available from: http://dx.doi.org/10.1086/677161.

15. Cockerill FR, Wilson JW, Vetter EA, Goodman KM, Torgerson CA, Harmsen WS, et al. Optimal Testing Parameters for Blood Cultures. Clinical Infectious Diseases. 2004 Jun 15;38(12):1724-30. Available from: http://dx.doi.org/10.1086/421087.

16. Bouza $E$, Sousa D, Munoz $P$, Rodriguez-Creixems $M$, Fron C, Lechuz JG. Bloodstream Infections: A Trial of the Impact of Different Methods of Reporting Positive Blood Culture Results. Clinical Infectious Diseases. 2004 Oct 15;39(8):1161-9. Available from: http://dx.doi.org/10.1086/424520.

17. Argemi $X$, Riegel P, Lavigne T, Lefebvre N, Grandpré N, Hansmann $Y$, et al. Implementation of Matrix-Assisted Laser Desorption Ionization-Time of Flight Mass Spectrometry in Routine Clinical Laboratories Improves Identification of Coagulase-Negative Staphylococci and Reveals the Pathogenic Role of Staphylococcus lugdunensis. Patel R, editor. Journal of Clinical Microbiology. 2015 Apr 15;53(7):2030-6. Available from: http://dx.doi.org/10.1128/jcm.00177-15.

18. Chen Y, Porter V, Mubareka S, Kotowich L, Simor AE. Rapid Identification of Bacteria Directly from Positive Blood Cultures by Use of a Serum Separator Tube, Smudge Plate Preparation, and Matrix-Assisted Laser Desorption Ionization-Time of Flight Mass
Spectrometry. Burnham C-AD, editor. Journal of Clinical Microbiology. 2015 Jul 22;53(10):3349-52. Available from: http://dx.doi.org/10.1128/jcm.01493-15.

19. Menezes LC, Rocchetti TT, Bauab K de C, Cappellano P, Quiles MG, Carlesse F, et al. Diagnosis by real-time polymerase chain reaction of pathogens and antimicrobial resistance genes in bone marrow transplant patients with bloodstream infections. BMC Infectious Diseases. 2013;13(1):166. Available from: http://dx.doi.org/10.1186/1471-2334-13-166.

20. Harris DM, Hata DJ. Rapid identification of bacteria and candida using pna-fish from blood and peritoneal fluid cultures: a retrospective clinical study. Ann Clin Microbiol Antimicrob. 2013;12(1):2. Available from: http://dx.doi.org/10.1186/1476-0711-12-2.

21. Weinstein MP, Towns ML, Quartey SM, Mirrett S, Reimer LG, Parmigiani G, et al. The Clinical Significance of Positive Blood Cultures in the 1990s: A Prospective Comprehensive Evaluation of the Microbiology, Epidemiology, and Outcome of Bacteremia and Fungemia in Adults. Clinical Infectious Diseases. 1997 Apr 1;24(4):584-602. Available from: http://dx.doi.org/10.1093/clind/24.4.584.

22. Lemming L, Holt HM, Petersen IS, Østergaard C, Bruun B. Bactec 9240 blood culture system: to preincubate at $35^{\circ} \mathrm{C}$ or not? Clinical Microbiology and Infection. 2004 Dec;10(12):1089-91. Available from: http://dx.doi.org/10.1111/j.1469-0691.2004.00969.x.

23. Thomas MW, Chauvenet AR, O'Suoji C. Repeating blood cultures in neutropenic children with persistent fevers when the initial blood culture is negative. Pediatr Blood Cancer. 2013 Oct 30;61(2):194-194. Available from: http://dx.doi.org/10.1002/pbc.24834.

24. Zhuo C, Zhong N-S. Concerns About "Comparison of 2Blood Culture Media Shows Significant Differences in Bacterial Recovery for Patients on Antimicrobial Therapy."Clinical Infectious Diseases. 2013 Mar 13;56(12):1840-1. Available from: http://dx.doi.org/10.1093/cid/cit149.

25. Klingspor L, Muhammed SA, Özenci V. Comparison of the two blood culture systems, Bactec 9240 and BacT/Alert 3D, in the detection of Candida spp. and bacteria with polymicrobial sepsis. European Journal of Clinical Microbiology \& Infectious Diseases. 2012 Jun 6;31(11):2983-7. Available from: http://dx.doi.org/10.1007/s10096-012-1650-6. 